\title{
Luminosity measurement at CMS
}

\section{Jessica Lynn Leonard* on behalf of the CMS Collaboration DESY}

Zeuthen, Germany

E-mail: jessica. lynn.leonard@desy.de

The measurement of the luminosity delivered by the LHC is pivotal for several key physics analyses. During the first three years of running, tremendous steps forward have been made in the comprehension of the subtleties related to luminosity monitoring and calibration, which led to an unprecedented accuracy at a hadron collider. The detectors and corresponding algorithms employed to estimate online and offline the luminosity in CMS are described. Details are given concerning the procedure based on the Van der Meer scan technique that allowed a very precise calibration of the luminometers from the determination of the LHC beams parameters. What is being prepared in terms of detector and online software upgrades for the next LHC run is also summarized.

Technology and Instrumentation in Particle Physics 2014

2-6 June, 2014

Amsterdam, the Netherlands

* Speaker 


\section{Luminosity at CMS}

The Compact Muon Solenoid (CMS) [1] is one of two general purpose experiments at the Large Hadron Collider (LHC) [2] at CERN, Geneva, Switzerland. In any physics measurement, the luminosity plays a primary role, among other quantities such as detector acceptance, efficiency, and background subtraction. An accurate luminosity determination is therefore essential for the CMS physics program.

\section{Offline luminosity measurement}

\subsection{Pixel cluster counting method}

The offline luminosity measurement [3] makes use of the CMS silicon pixel tracker. The pixel detector consists of 3 layers in the barrel region and 2 layers at each endcap, with coverage to $|\eta|=2.5$. Each pixel cell is $100 \times 150 \mu \mathrm{m}^{2}$ in surface size. The pixel detector has a total of 66 million channels, with $96.3 \%$ of the channels always operational. The maximum readout rate is $100 \mathrm{kHz}$. At an instantaneous luminosity of $10^{34} \mathrm{~cm}^{-2} \mathrm{~s}^{-1}$, the full detector has an occupancy of below $0.1 \%$. To measure luminosity, the number of pixel clusters in the detector is counted. The high fraction of fully live channels, which minimizes variance in detector acceptance, and low occupancy at high event rate, which means count rates are linear with event rate, make it especially suited for use as a luminometer. The pixel tracker can only operate in stable running conditions, which means it cannot provide an online luminosity measurement. However, it is stable and precise and is therefore used as the reference luminometer for CMS.

\subsection{Van der Meer calibration scan results and uncertainties}

The method of calibrating the luminosity measurement, developed by Simon van der Meer, involves scanning the beams through each other and plotting the detector response as a function of the separation distance [4]. Several effects must be corrected for during the Van der Meer scan analysis. The beam separation distance is determined from the current in the corrector magnets. This must be compared with the measured luminous region using the CMS tracker. In addition, the beams can grow in size during the 30 minutes of the scan. However, this effect can be measured during the scan and corrected for easily if the growth is linear. The beams can also drift in the $x-y$ plane during the scan, and the beam position may be affected by beam-beam repulsive effects. These can also be measured and corrected for.

It is convenient to assume that the beams have a Gaussian shape and that the shape is independent in the $\mathrm{x}$ and $\mathrm{y}$ directions. During the Van der Meer scan, the LHC setup is optimized to fulfill these conditions as much as possible. However, the exact functional form of the beam shape is unknown, and these assumptions currently contribute the largest component of the uncertainty to the luminosity measurement $(2 \%)$.

\subsection{Luminosity integration uncertainties}

Several effects must be accounted for during the analysis. The relationship between number of pixel clusters and luminosity can become nonlinear when pixel clusters belong to 


\begin{tabular}{|c|c|c|c|}
\hline & Systematic & correction (\%) & uncertainty (\%) \\
\hline \hline \multirow{4}{*}{ Integration } & Stability & - & 1 \\
\cline { 2 - 4 } & Dynamic inefficiencies & - & 0.5 \\
\cline { 2 - 4 } & Afterglow & $\sim 2$ & 0.5 \\
\hline \multirow{4}{*}{ Normalization } & Fit model & - & 2 \\
\cline { 2 - 4 } & Beam current calibration & - & 0.3 \\
\cline { 2 - 4 } & Ghosts and satellites & -0.4 & 0.2 \\
\cline { 2 - 4 } & Length scale & -0.9 & 0.5 \\
\cline { 2 - 4 } & Emittance growth & -0.1 & 0.2 \\
\cline { 2 - 4 } & Orbit Drift & 0.2 & 0.1 \\
\cline { 2 - 4 } & Beam-beam & 1.5 & 0.5 \\
\cline { 2 - 4 } & Dynamic- $\beta$ & - & 0.5 \\
\hline \hline & Total & & 2.5 \\
\hline
\end{tabular}

Table 1: Summary of the systematic uncertainties on the offline luminosity measurement, including both integration (luminometers) and normalization (calibration). The total offline luminosity uncertainty is $2.5 \%$.

more than one track. However, the loss of linearity was determined to be very small, only $1 \%$ at a pileup value of 200. The detector can also be affected by "dynamic inefficiencies" when the data rate becomes very high, causing the data acquisition system to become busy and preventing data taking, as shown in Figure 1. This effect is in general quite small, less than $0.5 \%$ overall. In addition, "afterglow" due to detector material activation can cause out-of-time response in the pixel cluster count. The afterglow effect was modeled assuming an exponential decay, and the effect was determined to be $\sim 2 \%$. In general, the uncertainty of the luminosity integration is low, contributing a total of $1.2 \%$ on the overall uncertainty, which is $2.5 \%$. The uncertainties for the offline luminosity measurement are summarized in Table 1.

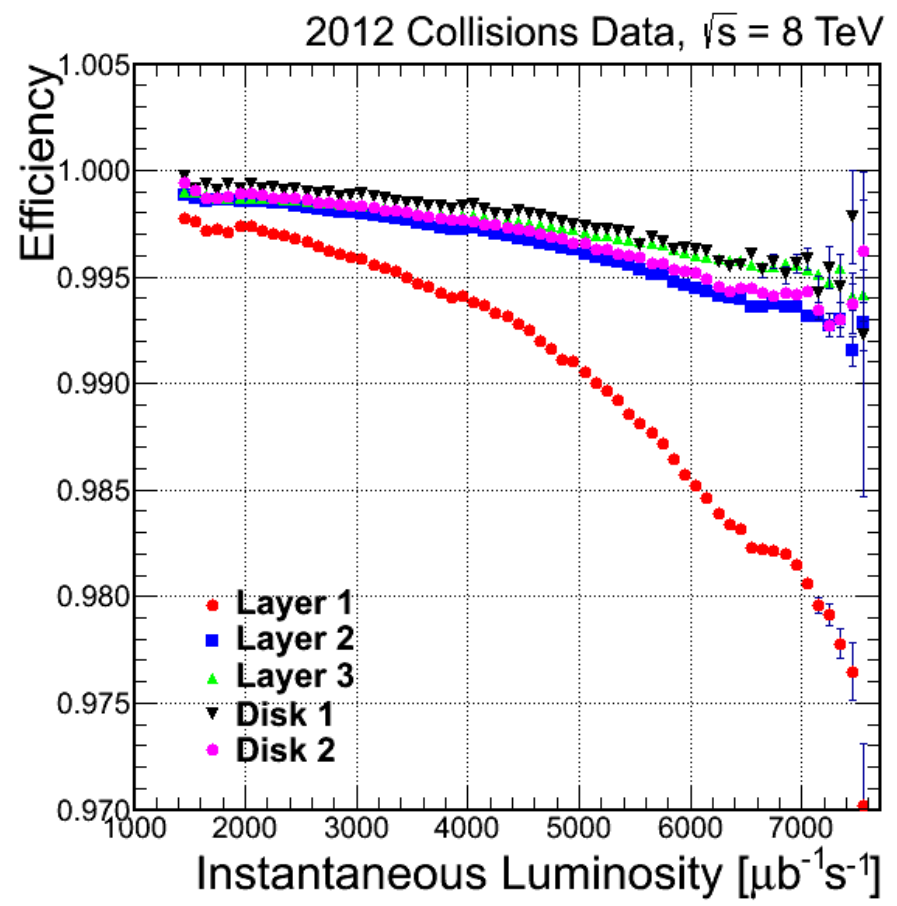

Figure 1: Dynamic inefficiencies occur when the data rate in the pixel detector becomes very high, causing the data acquisition system to become busy and preventing data taking. The effect is less than $0.5 \%$ overall. 


\subsection{Stability of measurement}

For an ideal luminometer, the luminosity calibration factor remains constant in time. Since it depends on the acceptance and efficiency for a real luminometer, it may need to be corrected according to the measured acceptance and efficiencies. Using only channels that have been active for the full run mitigates this need to a certain extent, but there are many other effects that can play a role. One method of ensuring the calibration factor remains constant is to check the pixel layers against each other, as shown in Figure 2. The relative comparisons show stability at the $0.5 \%$ level. Another stability check is a comparison with the $Z \rightarrow \mu \mu$ cross section. By definition the true $Z \rightarrow \mu \mu$ cross section is constant with time and constant beam energy. Therefore, if a different cross section is measured with time, it can be corrected and the corrections also applied to the luminosity measurement. This study is in progress.

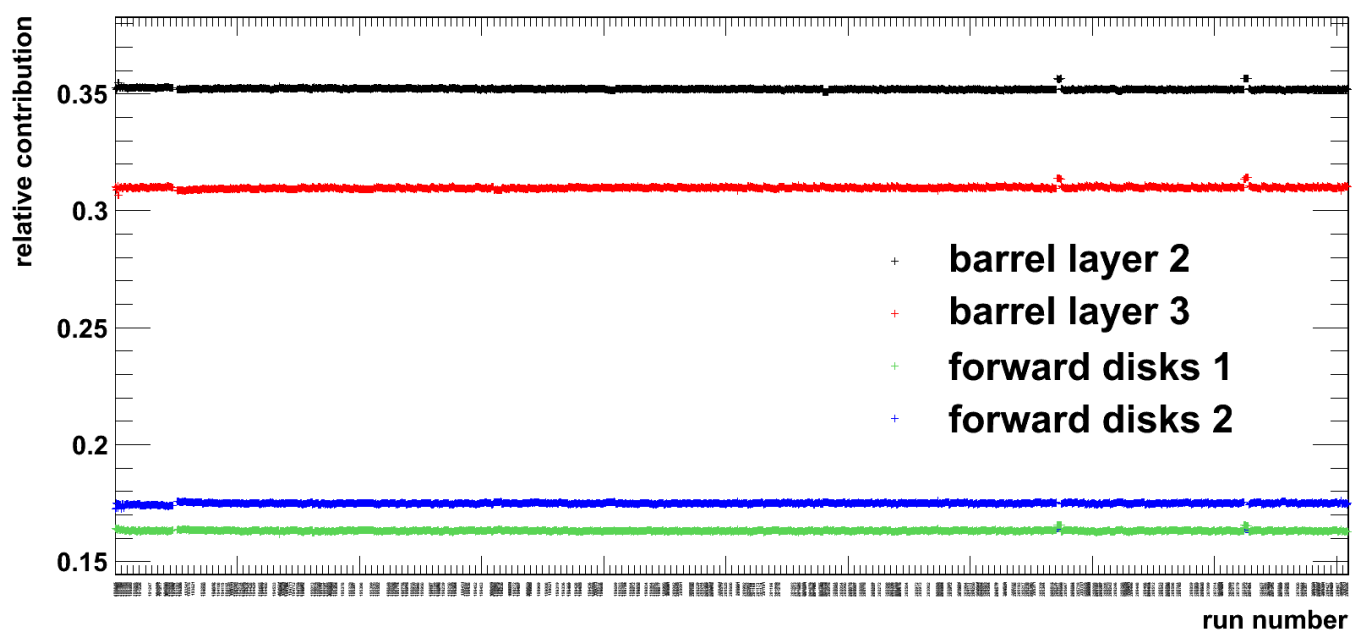

Figure 2: Relative contributions of the pixel layers are stable with time, indicating overall luminometer stability.

\section{Online luminosity measurement}

\subsection{Forward hadronic calorimeter zero-counting method}

For the online measurement [5], the forward hadronic calorimeter (HF) is used. HF is a sampling calorimeter made of quartz fibers embedded in an iron absorber. It covers the $|\eta|$ region from 3 to 5 , divided into 12 segments ("towers") in $\eta$ and 36 in $\phi$. The material allows fast collection of Cherenkov radiation ( $\sim 10 \mathrm{~ns})$. The quantity used to calculate the luminosity is the logarithm of the number of empty HF towers, which is linear at the occupancy level of normal running. Although the HF method is not as precise as the pixel cluster counting method, HF does not require stable running conditions and can therefore provide real-time bunch-bybunch luminosity under all beam conditions.

\subsection{Calibration results}

The online luminosity monitoring system also took part in the Van der Meer scans for the purpose of calibration. In the most recent scans (Jan/Feb 2013), the rate vs beam separation curves were fitted with a double Gaussian plus a constant. The double-Gaussian fit model 
provides the highest uncertainty in the online luminosity measurement (3\%), while beam-beam interaction effects on the beam position give the second highest (1.7\%). Overall, the 2013 set of scans provided a calibration of the online luminosity measurement with an uncertainty of $3.7 \%$.

\section{Upgrades to the luminosity system}

The current luminometers are undergoing upgrades to prepare for LHC Run II. The HF is getting new photo-multipliers to reduce spurious signals. The pixel detector will have four layers in the barrel and three in the endcap, covering a larger radial distance and providing a fourth space-point measurement over the full $\eta$ range of the pixel detector. In addition, new frontend electronics will improve high-rate performance.

Multiple new subsystems will be added into the online luminosity system, as well. This will introduce redundancy into the system, ensuring continuous performance in case one subsystem drops out, as well as providing confirmation of the measured luminosity value. The Fast Beam Condition Monitor (BCM1F), originally designed to provide online bunch-by-bunch measurements of the beam background, is being upgraded for inclusion into the luminosity monitoring system. It will consist of 24 single-crystal diamond sensors situated around the beam pipe on either side of the interaction point. The Pixel Luminosity Telescope (PLT), the only dedicated luminosity subsystem, will consist of 16 3-layer silicon pixel telescopes situated just beyond BCM1F. Each of the online luminosity subsystems will provide their raw data to the dedicated luminosity data acquisition system (LumiDAQ), which will collect and combine it for processing, publishing, and storage. The LumiDAQ system will also serve to synchronize the subsystems, providing identical timing and control signals, as well as providing counters to ensure proper resynchronization in case one system joins the run on-the-fly.

\section{Conclusion}

The CMS offline and online luminosity systems performed extremely well during LHC Run I, with total uncertainty values of $2.5 \%$ and $3.7 \%$ respectively. Both were calibrated using Van der Meer scans. The system was shown to be stable over a long period via comparisons between pixel layers. Preparations are underway for LHC Run II to improve the current devices and to include new online luminometers.

\section{References}

[1] The CMS Collaboration, The CMS experiment at the CERN LHC, J. Instrum., 3 (08):S08004, 2008.

[2] L. Evans and P. Bryant, LHC machine, J. Instrum., 3 (08):S08001, 2008.

[3] The CMS Collaboration, CMS Luminosity Based on Pixel Cluster Counting - Summer 2013 Update, CMS Public Analysis Summary CMS-PAS-LUM-12-001

[4] S. van der Meer, Calibration of the Effective Beam Height in the ISR, CERN-ISR-PO-68-31

[5] The CMS Collaboration, Luminosity measurement for the 2013 proton-ion run, CMS Public Analysis Sumary CMS-PAS-LUM-12-002 